\title{
The time to lead \\ ACRL seeks nominees for section offices
}

S

anding for ACRL section office is an opportunity to become involved with important issues affecting academic and research librarians in the 21st century, gain leadership experience, and build ties with colleagues. It is also an opportunity to be of service to your ACRL section community, ensuring that it continues to nurture the development of new members and forge deeper connections to the profession.

\section{ACRL section officers}

Candidates for vice-chair/chair elect, secretary, and members-at-large of ACRL sections are selected by the nominating committee of each section.

If you would like to nominate someone or be nominated yourself, contact the chair of the appropriate section nominating committee prior to the 2010 ALA Annual Conference in Washington, D.C. Terms of office will begin on July 1, 2011.

\section{1 section nominating committee chairs}

African American Studies Librarians: Vivian Njeri Fisher, Enoch Pratt Free Library, vfisher@prattlibrary.org

Anthropology and Sociology: Randy Hertzler, Montgomery College, randal. hertzler@montgomerycollege.edu

Arts: Bill Hemmig, Bucks County Community College, hemmigw@bucks.edu

Asian, African, and Middle Eastern: Bindu Bhatt, Columbia University, bb145@ columbia.edu
College Libraries: Alice Bahr, Salisbury University, ahbahr@salisbury.edu

Community College and Junior College Libraries: Contact CJCLS Vice-Chair David Wright, Sury Community College, wrightd@surry.edu

Distance Learning: Contact DLS ViceChair Robin Lockerby, National University, rlock@nu.edu

Education and Behavioral Sciences: Judy Walker, University of North CarolinaCharlotte, jwalker@uncc.edu

Instruction: Jean Caspers, Linfield College, jcaspers@linfield.edu

Law and Political Science: Contact LPSS Vice-Chair Chris Palazzolo, Emory University, cpalazz@emory.edu

Literatures in English: Steven Harris, University of New Mexico, srharris@unm.edu

Rare Books and Manuscripts: Mary Lacy, Library of Congress, mlac@loc.gov

Science and Technology: Virginia Ann Baldwin, University of Nebraska, vbaldwin2@ unl.edu

Slavic and East European: Cathy Zeljak, George Washington University, czeljak@ gwu.edu

University Libraries: Leslie Madden, Georgia State University, Imadden@gsu. edu

Western European Studies: Gerald Heverly, New York University, gerald.heverly@ nyu.edu

Women's Studies: Jennifer Gilley, Penn State University, jrg15@psu.edu $\boldsymbol{n}$ 